\title{
Surgical outcomes and survival for T4 gastric cancer extending to the transverse colon
}

\author{
Gang-Cheng Wang', Ying-Jun Liu', Chong-Qing Gao', You-Cai Wang', Hui-Fang Lv ${ }^{2}$, Bei-Bei Chen ${ }^{2}$, \\ Cai-Yun $\mathrm{Nie}^{2}$, Xiao-Bing Chen ${ }^{2}, \mathrm{Su}^{\mathrm{Xia}} \mathrm{Luo}^{2}$ \\ ${ }^{1}$ Department of General Surgery, ${ }^{2}$ Department of Gastrointestinal Medical Oncology, the Affiliated Cancer Hospital of Zhengzhou University, \\ Henan Cancer Hospital, Zhengzhou, China \\ Contributions: (I) Conception and design: YJ Liu, SX Luo; (II) Administrative support: SX Luo; (III) Provision of study materials: GC Wang, CQ \\ Gao; (IV) Collection and assembly of data: YC Wang, CY Nie; (V) Data analysis and interpretation: HF Lv, BB Chen; (VI) Manuscript writing: All \\ authors; (VII) Final approval of manuscript: All authors. \\ Correspondence to: Su-Xia Luo, MD; Ying-Jun Liu, MD. The Affiliated Cancer Hospital of Zhengzhou University, Henan Cancer Hospital, 127 \\ Dongming Road, Zhengzhou 450008, China. Email: zlyyluosuxia0361@zzu.edu.cn; yingjun6727@sina.com.
}

\begin{abstract}
Background: For the treatment of locally advanced (T4) gastric cancer, extended multi-organ resection remains controversial. This study aimed to evaluate the surgical outcomes and survival of patients with T4 gastric cancer extending to the transverse colon.

Methods: A total of 2,652 gastric cancer patients underwent surgery between December 2011 and December 2015. Data from 40 of these patients who underwent curative resection for $\mathrm{T} 4$ gastric cancer extending to the transverse colon were obtained. Patient characteristics, related complications, long-term survival, and prognostic factors for T4 gastric cancer were analyzed.

Results: Postoperative morbidity occurred in 5 (12.5\%) patients. All of the patients were cured with conservative treatment. No procedure-related mortality occurred. The 1-, 3-, and 5-year overall survival (OS) rates were $75.0 \%, 49.2 \%$, and $36.9 \%$, respectively, with a median survival time of 24 months. Univariate analysis revealed tumor size $(\mathrm{P}=0.049)$, advanced $\mathrm{T}$ stage $(\mathrm{P}=0.013)$, and lymph node metastasis $(\mathrm{P}=0.006)$ to be poor prognostic factors of OS. Advanced $\mathrm{T}$ stage and lymph node metastasis were identified by multivariate analysis as being independent prognostic factors. Further, it was observed that lymph node metastasis grade was associated with poorer OS.
\end{abstract}

Conclusions: Patients with T4 gastric cancer extending to the transverse colon might benefit from curative resection with acceptable morbidity and mortality.

Keywords: Stomach neoplasm; gastrectomy; prognosis

Submitted Feb 15, 2020. Accepted for publication Jun 23, 2020.

doi: $10.21037 /$ atm-20-3377

View this article at: http://dx.doi.org/10.21037/atm-20-3377

\section{Introduction}

Gastric cancer remains the third leading cause of cancerrelated mortality in the world, despite its incidence decreasing in recent decades (1). Currently, surgical resection is the most effective treatment for gastric cancer; however, once the tumor perforates the serosal layer and extends to the adjacent organs (T4), patients can expect a dismal prognosis. Multimodality therapy-surgical resection with perioperative chemotherapy or adjuvant chemoradiotherapy has been advocated for treatment of T4 gastric cancer. Despite this, for patients diagnosed with T4 gastric cancer, the 5-year overall survival (OS) rate is $<20 \%$ and approximately $30 \%$ for patients who undergo surgery (2). Such patients require radical gastrectomy with combined resection of the affected organs, which leads to increased perioperative morbidity and mortality. Therefore, selecting the appropriate surgical approach is pivotal for treating patients with $\mathrm{T} 4$ gastric cancer. 
The transverse colon has been reported as one of the organs most frequently involved in gastric cancer. Some studies reported the surgical outcomes and survival of T4 gastric cancer; however, $\mathrm{T} 4$ gastric cancer extending to the transverse colon has been the focus of few studies. In the present study, we evaluated the surgical outcomes and survival of 40 patients with $\mathrm{T} 4$ gastric cancer extending to the transverse colon.

We present the following article in accordance with the STROBE reporting checklist (available at http://dx.doi. org/10.21037/atm-20-3377).

\section{Methods}

A total of 2,652 patients with gastric cancer underwent surgery in the Department of General Surgery at our hospital in the period between December 2011 and December 2015. Our study focused on 40 of these patients who were diagnosed with $\mathrm{cT} 4 \mathrm{~b}$ gastric cancer extending to the transverse colon. The study was conducted in accordance with the Declaration of Helsinki. Approval for this study was received from the ethical committee of the hospital, and informed consent was obtained from all of the patients.

The inclusion criteria for the study were as follows: (I) aged 18-75 years; (II) transverse colon involvement; and (III) $\mathrm{R} 0$ resection performed. R0 resection was defined as complete tumor removal with no macroscopic or microscopic residual tumor tissue. Patients with peritoneal carcinomatosis and distant metastasis, and those who underwent resection of the distal esophagus and duodenum for expansion, splenectomy for lymph node dissection, or additional organ resections were excluded from the study.

All of the patients underwent gastrectomy with combined resection of the involved colon tissue. In curative resection cases, standard D2 lymph node dissections were performed. A distal subtotal or total gastrectomy was performed depending on the location of the primary tumor. A reconstruction using Roux-en- $Y$ was performed after a total gastrectomy; Roux-en-Y or Billroth II was used for subtotal gastrectomy. End-to-side anastomosis was utilized after a segmental colectomy. All of the patients were followed up once every three months for the first two years. At each follow-up visit, the patients underwent hematological tests and imaging examination [computed tomography (CT), magnetic resonance imaging, chest radiography, or ultrasonography]. After 2 years, the follow-up visits were extended to once every 6 months, and then after 5 years, to once every 12 months.

Histologic classification and staging were based on the eighth edition of the Union for International Cancer Control (UICC) tumor-node-metastasis (TNM) classification. Histologic differentiations were classified into well- and poorly-differentiated categories.

\section{Statistical analysis}

Continuous variables are expressed as the mean \pm standard deviation (SD). For qualitative variables, the chi-squared or Fisher's exact probability tests were performed. For continuous variables, Student's $t$-test was applied. Survival analysis was carried out using the Kaplan-Meier method and comparisons between the survival of the groups were performed using the log-rank test. Cox regression multivariate analysis was used to identify the independent prognostic factors of survival. A two-tailed $\mathrm{P}$ value $<0.05$ indicated statistical significance. Statistical analyses were performed using SPSS 16.0.

\section{Results}

Data from 40 patients who were diagnosed with cT4b gastric cancer extending to the transverse colon were analyzed. The tumors in 12 of these patients were located in the middle third of the stomach, 22 had tumors located in the lower third of the stomach, and 6 had tumors involving the entire stomach. The tumors ranged in diameter from 4-20 cm, with a mean diameter of $9 \mathrm{~cm}$. Neoadjuvant chemotherapy was received by 6 patients, and 31 patients received postoperative adjuvant chemotherapy.

Table 1 lists the clinicopathologic features of the 40 patients, of whom 29 were male and 11 were female. The patients had a mean age of 55.9 years, with a range between 42 and 75 years. Distal gastrectomies were performed on 15 patients, and total gastrectomies were performed on 25 patients. Invasion of the transverse colon (pT4b) was confirmed by histology in 22 of the 40 patients, whereas 18 exhibited only a desmoplastic reaction (pT4a). Lymph node metastases were detected in $29(72.5 \%)$ patients, and $12(30.0 \%)$ had N1, $9(22.5 \%)$ had N2, and 8 (20.0\%) had $\mathrm{N} 3$ disease. Histologic evaluation revealed 33 patients to have poorly-differentiated tumors, and the tumors of the remaining 7 were defined as well differentiated.

Postoperative complications occurred in 5 (12.5\%) patients, these were: anastomotic leakage (2 cases); intra-abdominal infection (1 case); massive hydrothorax 
Table 1 Clinicopathologic features

\begin{tabular}{lc}
\hline Features & Value \\
\hline Mean age (years) & 55.9 \\
Sex (male/female) & $29 / 11$ \\
Tumor size (cm) & $9.0 \pm 5.0$ \\
Tumor location & \\
Middle & 12 \\
Lower & 22 \\
Whole & 6 \\
Histologic type & \\
Well differentiated & 7 \\
Poorly differentiated & 33 \\
Depth of invasion & \\
T4a & 18 \\
T4b & 22 \\
Lymph node involvement & \\
Negative & \\
Positive & \\
III & \\
Noscular invasion & \\
\hline Yes & \\
\hline
\end{tabular}

( 1 case); and lung infection ( 1 case). All of the patients were cured with conservative treatment. No procedure-related mortality occurred.

The median follow-up period was 19 months (range, 7-69 months). The 1-, 3-, and 5-year OS rates were 75.0\%, $49.2 \%$, and $36.9 \%$, respectively, with a median survival time of 24 months. Univariate analysis revealed tumor size $(>9 \mathrm{~cm}$ ), advanced $\mathrm{T}$ stage, and lymph node metastasis to be associated with poor survival (Table 2). Multivariate analysis demonstrated that advanced $\mathrm{T}$ stage and lymph node metastasis were independent prognostic factors of OS
Table 2 Univariate prognostic analysis of survival in 40 patients with T4 gastric cancer

\begin{tabular}{|c|c|c|c|}
\hline Features & $\mathrm{n}$ & 3-year survival rate $(\%)$ & $P$ value \\
\hline Age (years) & & & 0.555 \\
\hline$\leq 60$ & 27 & 48.6 & \\
\hline$>60$ & 13 & 53.8 & \\
\hline Sex & & & 0.772 \\
\hline Male & 29 & 48.4 & \\
\hline Female & 11 & 45.5 & \\
\hline Tumor size (cm) & & & 0.049 \\
\hline$\leq 9.0$ & 25 & 58.4 & \\
\hline$>9.0$ & 15 & 41.7 & \\
\hline Histologic type & & & 0.638 \\
\hline Well differentiated & 7 & 47.6 & \\
\hline Poorly differentiated & 33 & 49.4 & \\
\hline Depth of invasion & & & 0.013 \\
\hline pT4a & 18 & 77.8 & \\
\hline pT4b & 22 & 26.5 & \\
\hline Lymph node metastasis & & & 0.006 \\
\hline No & 11 & 77.8 & \\
\hline Yes & 29 & 38.0 & \\
\hline \multicolumn{3}{|c|}{ Grade of lymph node metastasis } & 0.027 \\
\hline $\mathrm{pN} 1$ & 12 & 49.5 & \\
\hline $\mathrm{pN} 2-\mathrm{N} 3$ & 17 & - & \\
\hline Lymphatic invasion & & & 0.938 \\
\hline Yes & 30 & 47.4 & \\
\hline No & 10 & 58.3 & \\
\hline Vascular invasion & & & 0.301 \\
\hline Yes & 9 & - & \\
\hline No & 31 & 54.1 & \\
\hline
\end{tabular}

(Table 3). For patients with pT4a tumors, the overall 3-year survival rate was $77.8 \%$, whereas for patients with $\mathrm{pT} 4 \mathrm{~b}$ tumors, the overall 3 -year survival rate was $26.5 \%(\mathrm{P}=0.013$; Figure 1). Patients with N0 had a 3 -year survival rate of $77.8 \%$, which was significantly better than patients with $\mathrm{N}+(\mathrm{P}=0.006$; Figure 2). Patients at pN2 stage or higher had significantly poorer survival at 3 years than patients at $\mathrm{pN} 1$ stage $(\mathrm{P}=0.027$; Figure 3$)$. 
Table 3 Multivariate analysis of survival in 40 patients with T4 gastric cancer

\begin{tabular}{|c|c|c|c|c|}
\hline Variable & Regression coefficient & SE & Hazard ratio (95\% Cl) & $P$ value \\
\hline Depth of invasion & 1.294 & 0.606 & $3.646(1.112-11.955)$ & 0.033 \\
\hline Lymph node metastasis & 1.825 & 0.789 & $6.200(1.319-29.136)$ & 0.021 \\
\hline
\end{tabular}

SE, standard error.

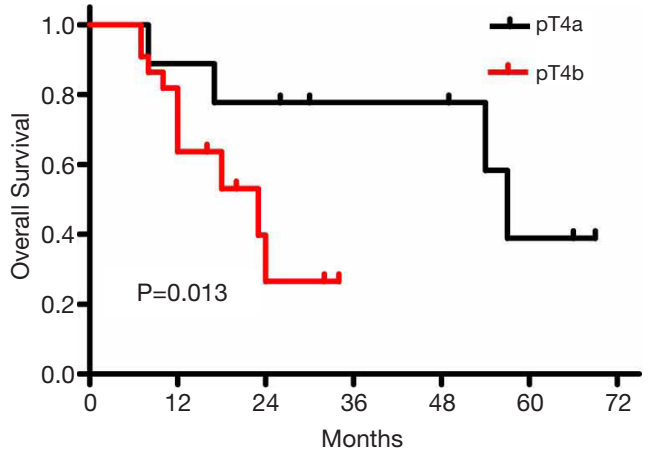

Figure 1 Overall survival based on T stage.

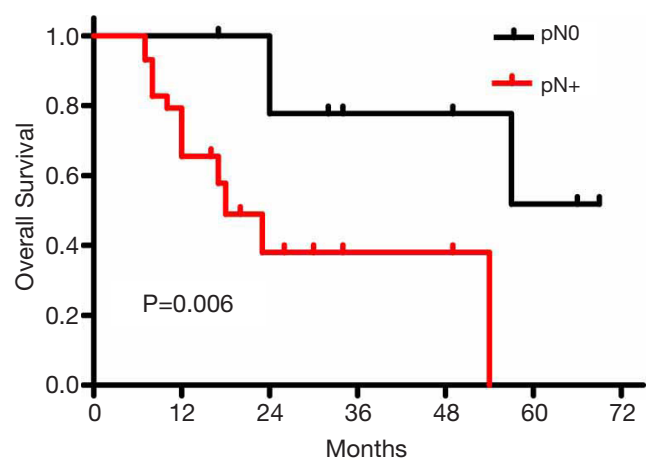

Figure 2 Overall survival based on $\mathrm{N}$ stage.

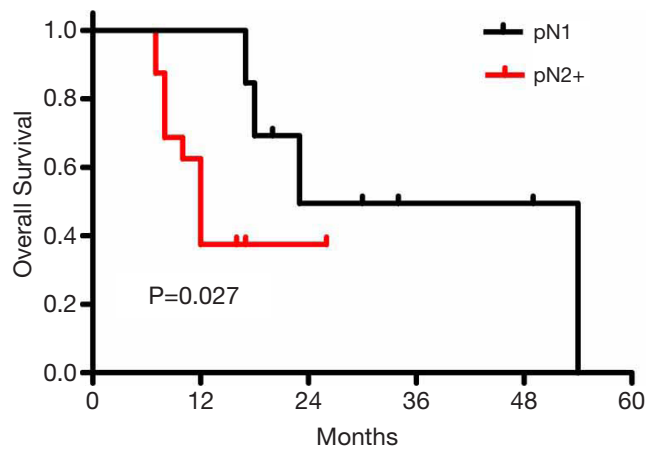

Figure 3 Overall survival based on the extent of lymph node metastasis.

\section{Discussion}

Gastric cancer is one of the most common cancers worldwide. In approximately $10 \%$ of patients, the gastric tumors perforate the serosa and extend to the adjacent organs $(3,4)$. The transverse colon is among the organs most likely to be affected by gastric cancer; however, it is still unknown whether patients with tumors invading the colon have improved survival compared with patients whose tumors invade other organs. Some studies have reported that the survival rate is not dependent on which organ is invaded (5-7). Pacelli et al. also found that patients with colon invasion had no survival advantage over those with other organs invaded (8). Although Dhar et al. reported that patients with colon or mesocolon invasion had better survival rates than patients with other organ invasion based on univariate analysis, their multivariate analysis found that invasion of the colon or mesocolon was not a significant factor of survival (9). These results indicate that the prognosis of patients with invasion to the transverse colon is comparable to that of patients who experience invasion in other organs.

In our study, the 5 -year OS rate was $36.9 \%$, with a complication rate of $12.5 \%$, which is considered acceptable. Fukuda et al. reported that the 5-year survival rate in T4 gastric cancer patients undergoing curative gastrectomy was $34.1 \%$ and the morbidity rate was $26.8 \%$ (4). Brar et al., who reviewed a total of 17 studies involving 1,343 patients with locally advanced gastric cancer, observed that the 5 -year survival rate after R0 resection was $32-35 \%$ and the overall complication rates ranged from $11.8-90.5 \%$ (2). In a recent study, a 3-year survival rate of $47.7 \%$ and a complication rate of $37.9 \%$ were obtained from patients with clinical $\mathrm{T} 4 \mathrm{~b}$ gastric cancer (10). Thus, the long-term outcomes in our study were similar to those in previous studies. However, we achieved a lower complication rate compared with previous studies, which could be attributed to the fact only colon resection was included in our study. Kasakura et al. found that patients who had additional organs resected had a higher 
complication rate compared with patients who underwent gastrectomy alone (11), and Ozer et al. found that patients who underwent MVR with two or more organs exhibited higher surgical morbidity (12). Based on these findings, we propose that extended gastrectomy with resection of the involved colon can be performed with minimal morbidity and can increase the odds of OS for patients with T4 gastric cancer extending to the transverse colon.

The most commonly reported prognostic factors of $\mathrm{T} 4$ gastric cancer patients are curability, the depth of tumor invasion, and lymph node metastasis. Curative resection offers the likelihood for a cure, and non-curative resection is usually adopted in patients with peritoneal carcinomatosis and distant metastasis as a palliative approach. In patients who underwent multivisceral resection without complete resection, survival was demonstrated to be significantly diminished compared to patients who received $\mathrm{R} 0$ resection $(13,14)$. Dhar et al. treated $150 \mathrm{~T} 4$ gastric cancer patients and reported that curative patients had survival benefit over non-curative patients (9). Furthermore, Mita et al. concluded that extended multi-organ resection could be beneficial only if curative surgery was performed (15). Therefore, our study was limited to patients without distant metastasis who were treated with curative resection. Our results also demonstrated that $\mathrm{R} 0$ resection resulted in favorable survival.

The present study determined advanced $\mathrm{T}$ stage to be an unfavorable prognostic factor for OS. In fact, even identifying $\mathrm{T}$ stage is challenging when the tumors extend to the adjacent organs. Fukagawa $e t$ al. found that the positive predictive values of preoperative computed tomographic scans in assessing T4 stage were low (16). Furthermore, intraoperative assessment of true invasion into adjacent organs may also present a challenge. Mita et al. reported that 19 of $41(46.3 \%) \mathrm{T} 4$ gastric cancer patients in their study had pathologically confirmed inflammatory adhesions (15). Similarly, $45 \%$ of the patients in our study had tumor adhesions to the colon (pT4a) rather than invasion of the colon (pT4b). When patients have $\mathrm{pT} 4 \mathrm{a}(\mathrm{cT} 4 \mathrm{~b})$ gastric cancer, it is difficult to determine if the affected organs require resection. In a previous study, Cheng et al. treated 179 patients with T4 gastric cancer, observing that combined resection achieved a better survival whether or not tumor adhesion or invasion had occurred in the adjacent organs (17). Therefore, extended multiorgan resection is recommended for curative resection in patients with $\mathrm{T} 4$ gastric cancer.

Lymph node metastasis is common in $\mathrm{T} 4$ gastric cancer. The lymph node metastasis rate in the current study was $72.5 \%$. Lymph node metastasis is a commonly reported prognostic factor of poor outcome in patients with T4 gastric cancer. Ozer et al. reported that lymph node metastasis is an independent poor prognostic factor in patients with locally advanced gastric cancer (12). Jeong et al. revealed that lymph node metastasis (higher than $\mathrm{pN} 3$ ) is an independent prognostic factor of poor prognosis in patients with $\mathrm{T} 4$ gastric carcinoma who underwent curative surgery (18). Further, it was observed that patients with extensive lymph node metastasis (N2 or N3) had a significantly poorer prognosis compared to patients with limited lymph node metastasis (N0 or N1) $(4,7)$. T4 gastric cancer with $\mathrm{N} 2$ or $\mathrm{N} 3$ nodal disease was considered as incurable by Cheng et al. and a contraindication for extensive surgery (17). In accordance with previous observations, lymph node metastasis, as well as lymph node metastasis grade, were associated with poorer overall survival in the patients in our study. We recommend performing extended multi-organ resection in $\mathrm{T} 4$ gastric cancer patients with limited lymph node metastasis.

The application of neoadjuvant therapy is increasingly advocated for patients with locally advanced gastric cancer. In the MAGIC randomized trial, 503 patients with gastroesophageal cancer received either perioperative chemotherapy combined with surgery ( $\mathrm{n}=250$ patients) or surgery alone ( $\mathrm{n}=253$ patients) (19). In this study, compared with surgery alone, perioperative chemotherapy and surgery improved overall survival and local control. In the French FFCD 9703 multicenter phase-III trial (20), 224 patients with resectable adenocarcinoma of the lower esophagus, the gastroesophageal junction, or the stomach were randomly assigned to receive surgery and perioperative chemotherapy or surgery alone. Higher R0 resection rates and improved overall survival were achieved in the perioperative chemotherapy group compared with the surgery alone group. However, multivariate analysis showed perioperative chemotherapy to have no significant effect for patients with gastric cancer. Another randomized trial, EORTC 40954, which involved 144 patients with locally advanced adenocarcinoma of the stomach or esophagogastric junction showed that neoadjuvant chemotherapy increased the $\mathrm{R} 0$ resection rate but failed to improve overall survival compared with surgery alone (21). The aforementioned studies demonstrate that neoadjuvant chemotherapy can decrease $\mathrm{T}$ and $\mathrm{N}$ stage and increase the $\mathrm{R} 0$ resection rate, whereas the survival benefit from neoadjuvant chemotherapy in distal gastric cancer 
remains indeterminate. A retrospective analysis from the National Cancer Database (1998-2011) indicated that neoadjuvant therapy may facilitate improved overall survival in patients with T4 gastric cancer (22). However, only $61.8 \%(648 / 1,049)$ of the patients who underwent surgical resection received R0 resection. Furthermore, it was not described whether D2 or D1 lymph node dissection was performed. Thus, more randomized controlled trial (RCT) studies are needed to demonstrate the survival benefit of neoadjuvant therapy in distal gastric cancer.

This study had some limitations, including the small number of patients in the sample. Moreover, the present study was a retrospective, single-center research projec. Further investigation with large sample sizes and randomized controlled studies are required to document the benefits of curative resection for the treatment of $\mathrm{T} 4$ gastric cancer extending to the transverse colon.

\section{Conclusions}

In conclusion, patients with $\mathrm{T} 4$ gastric cancer extending to the transverse colon might benefit from curative resection with acceptable morbidity and mortality.

\section{Acknowledgments}

Funding: None.

\section{Footnote}

Reporting Checklist: The authors have completed the STROBE reporting checklist. Available at http://dx.doi. org/10.21037/atm-20-3377

Data Sharing Statement: Available at http://dx.doi. org/10.21037/atm-20-3377

Conflicts of Interest: All authors have completed the ICMJE uniform disclosure form (available at http://dx.doi. org/10.21037/atm-20-3377). The authors have no conflicts of interest to declare.

Ethical Statement: The authors are accountable for all aspects of the work in ensuring that questions related to the accuracy or integrity of any part of the work are appropriately investigated and resolved. The study was conducted in accordance with the Declaration of Helsinki (as revised in 2013) and was approved by the institutional review board of the Affiliated Cancer Hospital of Zhengzhou University (No. 202005). Written informed consent was obtained from all of the patients.

Open Access Statement: This is an Open Access article distributed in accordance with the Creative Commons Attribution-NonCommercial-NoDerivs 4.0 International License (CC BY-NC-ND 4.0), which permits the noncommercial replication and distribution of the article with the strict proviso that no changes or edits are made and the original work is properly cited (including links to both the formal publication through the relevant DOI and the license). See: https://creativecommons.org/licenses/by-nc-nd/4.0/.

\section{References}

1. Global Burden of Disease Cancer Collaboration, Fitzmaurice C, Allen C, et al. Global, regional, and national cancer incidence, mortality, years of life lost, years lived with disability, and disability-adjusted life-years for 32 cancer groups, 1990 to 2015: a systematic analysis for the global burden of disease study. JAMA Oncol 2017;3:524-48.

2. Cunquero-Tomás AJ, Ortiz-Salvador JM, Iranzo V, et al. Sweet syndrome as the leading symptom in the diagnosis of gastric cancer. Chin Clin Oncol 2018;7:11.

3. Carboni F, Lepiane P, Santoro R, et al. Extended multiorgan resection for T4 gastric carcinoma: 25 -year experience. J Surg Oncol 2005;90:95-100.

4. Zubarayev M, Min EK, Son T. Clinical and molecular prognostic markers of survival after surgery for gastric cancer: tumor-node-metastasis staging system and beyond. Transl Gastroenterol Hepatol 2019;4:59.

5. Kunisaki C, Akiyama H, Nomuara M, et al. Surgical outcomes in patients with T4 gastric carcinoma. J Am Coll Surg 2006;202:223-30.

6. Zhang CD, Yamashita H, Seto Y. Gastric cancer surgery: historical background and perspective in Western countries versus Japan. Ann Transl Med 2019;7:493.

7. Isozaki H, Tanaka N, Tanigawa N, et al. Prognostic factors in patients with advanced gastric cancer with macroscopic invasion to adjacent organs treated with radical surgery. Gastric Cancer 2000;3:202-10.

8. Pacelli F, Cusumano G, Rosa F, et al. Multivisceral resection for locally advanced gastric cancer: an Italian multicenter observational study. JAMA Surg 2013;148:353-60.

9. Dhar DK, Kubota H, Tachibana M, et al. Prognosis of $\mathrm{T} 4$ gastric carcinoma patients: an appraisal of aggressive 
surgical treatment. J Surg Oncol 2001;76:278-82.

10. Mita K, Ito H, Katsube T, et al. Prognostic Factors Affecting Survival After Multivisceral Resection in Patients with Clinical T4b Gastric Cancer. J Gastrointest Surg 2017;21:1993-9.

11. Kasakura Y, Fujii M, Mochizuki F, et al. Is there a benefit of pancreaticosplenectomy with gastrectomy for advanced gastric cancer? Am J Surg 2000;179:237-42.

12. Ozer I, Bostanci EB, Orug T, et al. Surgical outcomes and survival after multiorgan resection for locally advanced gastric cancer. Am J Surg 2009;198:25-30.

13. Kim DY, Joo JK, Seo KW, et al. T4 gastric carcinoma: the benefit of non-curative resection. ANZ J Surg 2006;76:453-7.

14. Xiao L, Li M, Xu F, et al. Extended multi-organ resection for cT4 gastric carcinoma: A retrospective analysis. Pak J Med Sci 2013;29:581-5.

15. Mita K, Ito H, Fukumoto M, et al. Surgical outcomes and survival after extended multiorgan resection for $\mathrm{T} 4$ gastric cancer. Am J Surg 2012;203:107-11.

16. Fukagawa T, Katai H, Mizusawa J, et al. A prospective multi-institutional validity study to evaluate the accuracy of clinical diagnosis of pathological stage III gastric cancer (JCOG1302A). Gastric Cancer 2018;21:68-73.

17. Cheng CT, Tsai CY, Hsu JT, et al. Aggressive surgical

Cite this article as: Wang GC, Liu YJ, Gao CQ, Wang YC, Lv HF, Chen BB, Nie CY, Chen XB, Luo SX. Surgical outcomes and survival for $\mathrm{T} 4$ gastric cancer extending to the transverse colon. Ann Transl Med 2020;8(15):947. doi: 10.21037/atm-20-3377 approach for patients with T4 gastric carcinoma: promise or myth? Ann Surg Oncol 2011;18:1606-14.

18. Jeong O, Choi WY, Park YK. Appropriate selection of patients for combined organ resection in cases of gastric carcinoma invading adjacent organs. J Surg Oncol 2009;100:115-20.

19. Cunningham D, Allum WH, Stenning SP, et al. Perioperative chemotherapy versus surgery alone for resectable gastroesophageal cancer. $\mathrm{N}$ Engl J Med 2006;355:11-20.

20. Ychou M, Boige V, Pignon JP, et al. Perioperative chemotherapy compared with surgery alone for resectable gastroesophageal adenocarcinoma: an FNCLCC and FFCD multicenter phase III trial. J Clin Oncol 2011;29:1715-21.

21. Schuhmacher C, Gretschel S, Lordick F, et al. Neoadjuvant chemotherapy compared with surgery alone for locally advanced cancer of the stomach and cardia: European Organisation for Research and Treatment of Cancer randomized trial 40954. J Clin Oncol 2010;28:5210-8.

22. Lowenfeld L, Datta J, Lewis RS Jr, et al. Multimodality treatment of T4 gastric cancer in the United States: utilization trends and impact on survival. Ann Surg Oncol 2015;22 Suppl 3:S863-72. 